\title{
Bronchial wash Gene Xpert MTB/RIF in lower lung field tuberculosis: Sensitive, superior, and rapid in comparison with conventional diagnostic techniques
}

\author{
Shital Patil ${ }^{1}$, Swati Narwade ${ }^{2}$, Mazhar Mirza ${ }^{3}$ \\ 'Department of Pulmonary Medicine, MIMSR Medical College, Latur, India; \\ 2Department of Obstetrics \& Gynecology, Government Medical College, Latur, India; \\ ${ }^{3}$ Department of Internal Medicine, MIMSR Medical College, Latur, India
}

Address for Correspondence: Dr. Shital Patil, Department of Pulmonary Medicine, MIMSR Medical College, Latur, India.

Email: drsvpatil1980@gmail.com

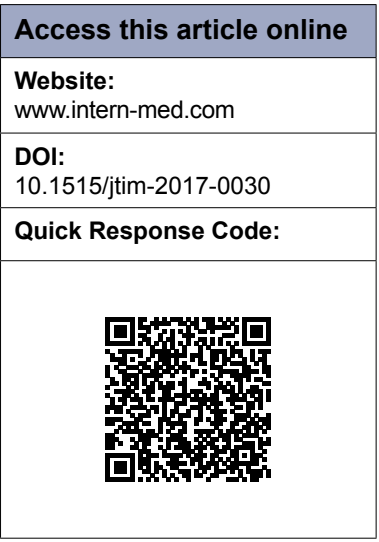

\section{ABSTRACT}

Background: Lower lung field tuberculosis (LLF TB) is an atypical presentation of tuberculosis (TB). LLF TB is common, and a proportionate number of non-resolving pneumonia cases are diagnosed to have pulmonary TB. Materials and Methods: The prospective observational study was conducted during June 2013 to December 2015 in the Department of Pulmonary Medicine, MIMSR Medical College, Latur, India; the objective of the study is clinical, microbiological, and radiological presentation of LLF TB and the comparison of yield of conventional diagnostic techniques and bronchoscopy guided modalities in LLF TB. Additional important objective of the study is to find LLF TB in patients with nonresolving pneumonia (NRP). A total of 2,600 patients with pulmonary TB were included in the study after inclusion and exclusion criteria. Ethical clearance was taken from the ethical committee of the institutional review board. Consent was taken from the patients before inclusion in the study. Statistical analysis was done using chisquare test. Results: In the present study, $300(11.53 \%)$ cases of LLF TB of total 2600 pulmonary tuberculosis were included, females constitutes $66.66 \%$ (200/300) with mean age of $58.4 \pm 11.8$ years and males constitutes $33.34 \%(100 / 300)$ with mean age of $56.8 \pm 10.6$ years. Constitutional symptoms were observed as cough in $93 \%$ cases, fever in $83 \%$ cases, shortness of breath in $72 \%$ cases, anorexia in $91 \%$ cases, and weight loss in $84 \%$ cases. Radiological assessment of study cases documented the involvement of right lower zone in $84 \%$ cases and left lower zone in only $16 \%$ cases. In the studied LLF TB cases, 57 cases $(20.66 \%)$ were diagnosed by routine sputum microscopic examination for acid fast bacilli (AFB) and 80 cases (28\%) were diagnosed by induced sputum microscopic examination for AFB. In the study of 170 LLF TB cases, headto-head comparison between conventional diagnostic techniques (sputum microscopy and Induced sputum microscopy for AFB) made diagnosis in 60 cases, while bronchoscopy-guided sampling techniques (BAL for AFB and BAL for Gene Xpert MTB/RIF) made diagnosis in 155 cases $(91.17 \%)(P<0.00001)$. Comorbid conditions such as human immunodeficiency virus (HIV) coinfection in 36 cases $(12.00 \%)$, Diabetes mellitus in 64 cases $(21.33 \%)$, and chronic kidney disease (CKD) in 22 cases (7.33\%) were observed. Comorbidities were observed in $41.67 \%$ of the studied cases and found very significant assessment to have successful treatment outcome $(P<0.00001)$. In the study of 300 LLF TB cases, 60 cases were having NRP pattern. In LLF TB cases with NRP pattern, bronchoscopy-guided bronchial wash microscopy for AFB made diagnosis in 18 cases $(42 \%)$, while bronchoscopy-guided BAL for Gene Xpert MTB/RIF made diagnosis in 58 cases $(96.66 \%)(P<0.00001)$. Conclusion: LLF TB is usually underdiagnosed because of diverse clinical and radiological presentation, less diagnostic yield of conventional diagnostic modalities, and these modalities used routinely and universally. Bronchoscopy-guided diagnostic techniques are superior, sensitive, and reliable to confirm LLF TB. Gene Xpert MTB/ RIF in bronchial wash samples is found to be best diagnostic modality in evaluating LLF TB and should be used routinely to have successful treatment outcome. A proportionate number of NRP cases are having LLF TB and a high index of suspicion is a must while evaluating these cases.

Key words: lower lung field tuberculosis (LLF TB), bronchoscopy, BAL Gene Xpert MTB/RIF, treatment outcome 


\section{INTRODUCTION}

Tuberculosis (TB) is an ancient disease affecting mankind described as far back as $10,000 \mathrm{BC}$, and it is still the major health problem worldwide. According to the World Health Organization (WHO), 9 million people fell ill with TB in 2013, including 1.1 million cases among people living with human immunodeficiency virus (HIV). ${ }^{[1]}$ In India, 8.7 million new cases of TB (13\% co-infected with HIV) and 1.4 million people died from TB, including almost 1 million deaths among HIV-negative individuals and 430,000 deaths among people who were HIV-positive, were estimated in the year 2011. ${ }^{[1]}$ In India, more than $40 \%$ is infected with TB and 1.9 million people developed TB every year. ${ }^{[1]}$ Though pulmonary TB commonly affects the upper lung fields, lower lung field tuberculosis (LLF TB) is also not uncommon. This often causes great confusion in the diagnosis. HIV/AIDS epidemic has considerably increased the incidence of middle and lower lung field tuberculosis (LLF TB) which is frequently associated with negative sputum smear because of lower bacillary load. ${ }^{[2]}$

LLF TB is defined ${ }^{[3]}$ as "tuberculosis disease found below an imaginary line traced across the hila and including the parahilar regions on a standard posterior-anterior chest roentgenogram." Ossen ${ }^{[4]}$ subdivided his cases into pure and impure groups: the pure group has no visible lesions in the upper lung fields and the impure group has nodular or fibrotic infiltrations in one or both apices. Other terms used for the same entity are "basal, lower lobe, hilar, parahilar, and perihilar tuberculosis." [3]

The most likely explanation for the development of LLF TB is transbronchial perforation of a hilar lymph node, with spread to the adjacent lung. ${ }^{[1,5]}$ Thus, lower lung field disease occurs as a continuation of the primary TB infection or soon afterwards in the post-primary period. ${ }^{\left[{ }^{[}\right]}$ This explanation is consistent with the high incidence of endobronchial involvement and with reported clinical and radiologic observations. ${ }^{[6]}$ A diagnosis of endobronchial disease is made when bronchoscopic evidence of stenosis or severe tracheobronchitis is detected or when there is roentgenographic evidence of atelectasis or tension cavities. ${ }^{[7]}$ Other mechanisms postulated in the pathogenesis of LLF TB are restricted ventilation, costal breathing, and retrograde lymphatic flow from involved hilar nodes. ${ }^{[5]}$ It does not appear that patients with lower lung field disease have especially lowered resistance to $\mathrm{TB}^{\left[{ }^{[7]}\right.}$ According to previous studies, the following conditions occur more frequently in patients with LLF TB than in general population with TB: diabetes mellitus, pregnancy, advanced age, malignancies, and advanced liver and renal diseases. ${ }^{[8,9]}$
Culture is the "gold standard" for final determination, but it is slow and may take up to $2-8$ weeks. Although smear microscopy for acid-fast bacilli (AFB) is rapid and inexpensive, it has poor sensitivity and a poor positive predictive value (PPV). Thus, rapid identification, which is essential for earlier treatment initiation, improved patient outcomes, and more effective public health interventions, relies on nucleic acid amplification techniques. The Gene Xpert MTB/RIF assay is a novel integrated diagnostic device that performs sample processing and heminested real-time polymerase chain reaction (PCR) analysis in a single hands-free step for the diagnosis of TB and rapid detection of Rifampicin resistance in clinical specimens. The MTB/RIF assay detects Mycobacterium tuberculosis and Rifampicin resistance by PCR amplification of the 81-bp fragment of the $r p o B$ gene of $M$. tuberculosis and subsequent probing of this region for mutations that are associated with rifampicin resistance. The assay can generally be completed in less than $2 \mathrm{~h} .{ }^{[1]}$

Richard Winterbauer et al. had empirically defined slowly resolving pneumonia in immune-competent patients as either less than $50 \%$ clearing at 2 weeks or less than complete clearance at 4 weeks in a patient who has defervesced and symptomatically improved with antibiotic therapy. ${ }^{[12]}$ Normal resolution of pneumonia is not easily defined. It can vary depending on the infecting organism and the host immune status. Patients typically note subjective improvement within 3-5 days of initiation of treatment. ${ }^{13}$ Nonresolving pneumonia (NRP) is defined as pneumonia with a slow resolution of radiographic infiltrates or clinical symptoms despite adequate antibiotic treatment (10-14 days). This can be due to defects in local or systemic immune defense mechanisms and due to the presence of unusual organism, resistant bacteria, or diseases that mimic pneumonia. ${ }^{[13]}$

In this study, we evaluated the role of bronchoscopy in the diagnosis of LLF TB with special emphasis on Gene Xpert MTB/RIF. We also compared the conventional tests such as sputum microscopy and induced sputum microscopy for AFB with bronchoscopy-guided bronchial wash for AFB microscopy and rapid nucleic acid amplification test, that is, Gene Xpert MTB/RIF.

\section{MATERIALS AND METHODS}

This is a prospective observational study conducted during June 2013 to December 2015 at the Department of Pulmonary Medicine, MIMSR Medical College, Latur, India, to study the clinical, microbiological, and radiological presentation of LLF TB and compare the yield of conventional diagnostic techniques and bronchoscopyguided modalities in LLF TB. Additional important 
objective of the study is to find LLF TB in cases with NRP. A total 2,600 patients with pulmonary TB were included in the study. Ethical clearance was taken from the ethical committee of the college. Consent from the patients was taken before inclusion in the study.

\section{Inclusion criteria}

1. Case definitions for pulmonary $\mathrm{TB}^{[10]}$ :

a. Pulmonary Tuberculosis, Smear-Positive-TB in a patient with at least one smear-positive for AFB out of the two initial sputum smear examination by direct microscopy. b. Pulmonary Tuberculosis, Smear-Negative-a patient with symptoms suggestive of $\mathrm{TB}$ with two smear examination negative for $\mathrm{AFB}$, with evidence of pulmonary TB by microbiological methods (culture positive or by other approved molecular methods) or chest X-ray is classified as having smear-negative pulmonary TB

2. Cases of diabetes mellitus, HIV, malignancies, and chronic kidney disease (CKD) with symptoms and radiological feature suggestive of $\mathrm{TB}$ are also included in study.

3. NRP cases after fulfilling definition. ${ }^{[13]}$

\section{Exclusion Criteria}

1. Age less than 12 years

2. Extra pulmonary TB cases

3. Pulmonary TB cases with pleural effusion, bilateral lung involvement, and concomitant upper lung field involvement are also excluded from the study.

\section{Methodology}

All the patients attending outdoor unit and admitted in indoor unit with complaints of cough, weight loss, fever, hemoptysis, and anorexia and history of contact with TB were enrolled in the study. Enrolled patients were also investigated for any systemic illness such as diabetes mellitus, chronic liver diseases, asthma, CKD, and HIV. Any relevant past history and personal history including dietary habits, smoking, alcohol, and other addictions were also taken.

LLF TB on chest X-ray was defined as the area lying below the horizontal arbitrary line drawn across the hila on the chest X-ray (PA film). Parahilar region was considered in the lower lung fields. ${ }^{[3]}$ Whenever necessary, disease located in the lower lung fields with doubtful costophrenic sulcus collection we performed lateral films. Record of the radiological reading in terms of consolidation, nodular opacity, and cavitation was also noted.

Diagnostic modalities were categorized as conventional in which routine sputum microscopy and induced sputum microscopy for AFB was done. Bronchoscopy-guided techniques were used as bronchial wash sample for AFB microscopy and bronchial wash specimen for Gene Xpert MTB/RIF.

Diagnosis of TB was made by sputum examination for AFB by Ziehl-Neelsen technique, for which two sputum samples were collected, of which one sample was collected preferably in the morning. Those patients who fail to produce sputum sample are advised for "induced sputum" sample.

\section{Procedure of induced sputum}

A brief description of the procedure was given to each patient. To avoid contamination, the patients were asked for rinsing and repeated gargling with tap water until the returned fluid was free from debris. About $20 \mathrm{~mL}$ of 3\% hypertonic saline was injected into the nebulizer reservoir device. The assembly was connected to the ultrasonic nebulizer. The procedure was carried out in a well-ventilated room with windows open and adequate staff protection measures were taken. The patients were instructed to inhale and exhale the mist of the nebulized solution through the mouth piece only. The inhalation of hypertonic saline was interrupted every $5 \mathrm{~min}$, so that the patient could expectorate the sputum into a clean sterile sputum container. The procedure was continued until an adequate amount of sputum sample $(3 \mathrm{~mL}$ or more) was obtained or for a maximum of $15 \mathrm{~min}$ has passed without success or the patients complained of shortness of breath or wheeze. The patient was closely monitored at all times during the procedure and $1 \mathrm{~h}$ after the procedure. The nebulizer equipment was decontaminated after each session by thorough washing and soaking in glutaraldehyde overnight. Diagnosis of TB was made by sputum examination for AFB by Ziehl-Neelsen technique.

Those cases negative for AFB after conventional sputum analysis and induced sputum examination were undergone bronchoscopy with Fujinon EPX-201H fiber-optic video bronchoscope. Bronchoscopy-guided bronchial wash collected with $60 \mathrm{~mL}$ saline instilled in middle and lower lobe segments, and two aliquots of bronchial wash samples were collected with suction during procedure. One bronchial wash sample is sent for AFB microscopy analysis and second sample for Gene Xpert MTB/RIF analysis. Bronchial wash specimens were processed, centrifuged, and the sediment were assessed for AFB microscopy. Bronchial wash second specimen was undergone cartridge-based test for Gene Xpert MTB/RIF analysis, in which first part is suggestive of MTB genome detection and second part is for $r p o B$ mutation. In single setting, Gene Xpert analysis made diagnosis of drug-sensitive or drug-resistant TB in $2 \mathrm{~h}$.

All the possible measures were taken to confirm the diagnosis of tuberculosis, and in case with high index of 
suspicion of TB, we have started anti-tuberculosis therapy (ATT), although results are not favoring the diagnosis. Decision to start ATT in these cases was supported by clinical and radiological response after 4 weeks of therapy, and if the response is satisfactory, we continued the therapy for 6 months. Decision to start ATT in these cases were clinician decision to treat, is one of the criteria in case definition of tuberculosis. As India is high TB-burden country, we offered without any confusion in these cases with, although all possible measures fail to detect $\mathrm{AFB}$ or genome of MTB.

All the study cases were offered standard chemotherapy as per national guidelines for anti-tuberculosis regimen containing four drugs, isoniazid, rifampicin, and pyrazinamide, are given for 2 months as intensive phase and isoniazid with rifampicin for 4 months as continuation phase to complete total 6 months.

The statistical analysis was done using chi-squared test (three methods of chi-squared test such as independence, goodness of fit, and proportion test). Significant values of $\chi 2$ were seen from probability table for different degree of freedom required. $P$ value was considered significant if it was below 0.05 and highly significant in case if it was less than 0.001 .

\section{RESULTS}

A total of 2,600 cases of pulmonary TB were initially screened and $300(11.53 \%)$ cases of LLF TB were enrolled after assessing inclusion and exclusion criteria. In study cases, predominant female gender distribution with $66.66 \%$ (200/300) of total study cases and males $33.34 \%$ (100/300). Mean age in female group is $58.4 \pm 11.8$ years and male group is $56.8 \pm 10.6$ years. Constitutional symptoms in study cases were cough in $93 \%$ cases, fever in $83 \%$ cases, shortness of breath in $72 \%$ cases, anorexia in $91 \%$ cases, and weight loss in $84 \%$ cases. Radiological assessment in study cases was observed as right lower zone in $84 \%$ cases and left lower zone in only $16 \%$ cases.

We observed 100 cases below 50 years of age, of whom 60 cases were female $(66.00 \%)$ and 40 were male $(40.00 \%)$, and 200 cases were above 50 years of age, of whom 120 cases were female $120(60.00 \%)$ and 80 were male (40.00\%) (Figure 1).

In all study cases of LLF TB, 57 cases (20.66\%) were diagnosed by routine sputum microscopic examination for AFB done on three samples collected all on early morning preferably fasting and 80 cases (28\%) were diagnosed by induced sputum microscopic examination for AFB. In the study of selected 170 LLF TB cases, bronchoscopy-guided samples made highly significant improvement $(P<0.00001)$ in diagnostic yield and made diagnosis in $155(91.17 \%)$ cases (Table 1).

In study cases of LLF TB, comorbid conditions such as HIV co-infection was in 36 cases (12.00\%), Diabetes mellitus in 64 cases $(21.33 \%)$, and CKD in 22 cases $(7.33 \%)$ were observed. Comorbidities were observed in $41.67 \%$ of study cases, while $59.33 \%$ cases were without any comorbidity and found very significant assessment to have successful treatment outcome. Pregnancy is an additional risk factor for LLF TB and documented in 9 cases. $(P<0.00001 ;$ Table 2$)$

In study of selected 170 LLF TB cases, head-to-head comparison between conventional diagnostic techniques (sputum microscopy and induced sputum microscopy for AFB) made diagnosis in 60 cases, while bronchoscopyguided sampling techniques (BAL for AFB and BAL for Gene Xpert MTB/RIF) made diagnosis in 155 cases. Bronchoscopy-guided samples found to be highly

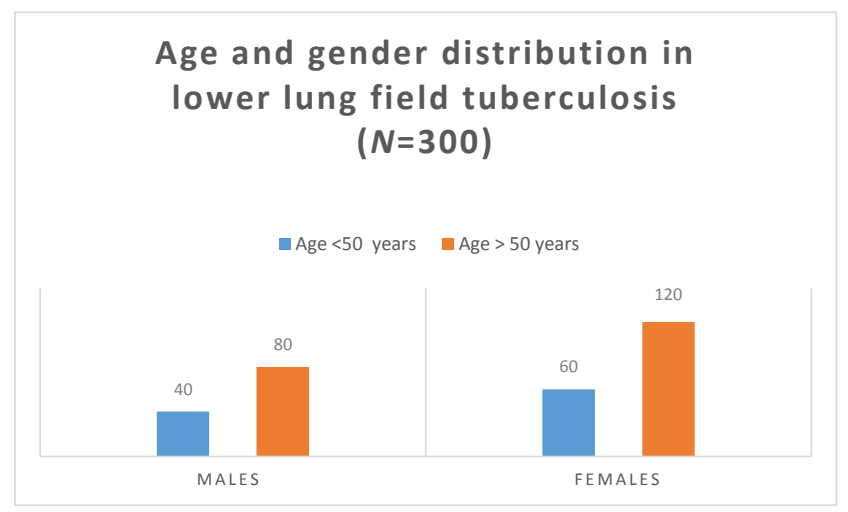

Figure 1: Bar diagram showing age and gender distribution in lower lung field tuberculosis

\begin{tabular}{lll}
\hline Table 1: Yield of diagnostic procedures in LLF TB & & \\
\hline Technique used for diagnosis & Number of patients $(\boldsymbol{n}=\mathbf{3 0 0})$ & Percentage (\%) \\
\hline Sputum microscopy for AFB & 57 & 20.66 \\
Induced sputum microscopy for AFB & 80 & 28.00 \\
Bronchial wash for microscopy & 98 & 32.66 \\
Bronchial wash for Gene Xpert MTB/Rif (performed in 170 cases) & 155 & 91.17 \\
\hline
\end{tabular}

LLF TB: lower lung field tuberculosis. Chi-square goodness of fit: $\chi^{2}=53.87, d f=3, P<0.00001$. 
significant $(P<0.00001)$ and superior to conventional diagnostic techniques (Table 3 ).

Sensitivity of Gene Xpert MTB/RIF in sputum-negative LLF TB in our study is $91.17 \%$. Culture of bronchoscopy sample for MTB was not done because of time factor, cumbersome, and cost effectiveness.

Analyzing Post hoc by odds ratio suggests that bronchoscopy-guided sampling techniques (bronchial wash for AFB and BAL Gene Xpert analysis) is 18.94 times superior to conventional investigation techniques (sputum smear microscopy and induced sputum microscopy for AFB) available to diagnose LLF TB (Table 4).

In the study of 300 LLF TB cases, 60 cases were having NRP pattern. In LLF TB cases with NRP pattern, bronchoscopy-guided bronchial wash microscopy for AFB made diagnosis in 18 cases (42\%), while bronchoscopyguided BAL for Gene Xpert MTB/RIF made diagnosis in 58 cases $(96.66 \%)$. BAL Gene Xpert MTB/RIF analysis is highly significant $(P<0.00001)$ and superior to BAL microscopy for AFB (Table 4).

Analyzing post hoc by odds ratio suggests that bronchoscopy-guided sample techniques including BAL for Gene Xpert MTB/RIF is 49.71 times superior to bronchial wash and also conventional investigation methods available to diagnose LLF TB in cluster of NRP cases (Table 4).

\section{DISCUSSION}

\section{Prevalence of LLF TB in study cases}

LLF TB was first reported by Kidd in $1886 .{ }^{(14)} \mathrm{A}$ total of 2,600 cases of pulmonary TB were initially screened and LLF TB was observed in $300(11.53 \%)$ cases. A review of literature shows a great variation in the reported frequency. It varies from $0.63 \%$ (Mathur et al.) ${ }^{[15]}$ to $6.4 \%$ (Vishwanathan). ${ }^{[16]}$ Incidence of LLF TB was observed by Zuber et al. ${ }^{[17]}$ as $10.6 \%$, Berger et al. ${ }^{[18]}$ as $7 \%$, Parmer et al. ${ }^{[19]}$ as $3.4 \%$, Hamilton et al. ${ }^{[20]}$ as $3 \%$, Chang et al. ${ }^{[9]}$ as $5.1 \%$, and Ross et al. ${ }^{[21]}$ as $18.3 \%$.

The discrepancies in the reported incidences of LLF TB may be due to confusion in the terms and definitions used, such as basal, lower lobe, or LLF TB. ${ }^{[2]}$ Also, the studies from TB sanatoria reported a lower percentage ${ }^{[19]}$ than general hospitals ${ }^{[9]}$ that may lack the bronchoscopy facility or underutilize the bronchoscopy-guided techniques for the evaluation of these cases. ${ }^{[0]}$

Most unique aspect of our study is that we performed bronchoscopy and evaluated the role of Gene Xpert MTB/RIF in cases "suspected of LLF TB" and in cases with presentation such as NRP and observed excellent role of these modalities in LLF TB rather than to start empirical treatment by considering them sputumnegative case of TB.

\begin{tabular}{lll}
\hline \multicolumn{2}{l}{ Table 2: Comorbid conditions and LLF TB $(\boldsymbol{n}=\mathbf{3 0 0})$} & \\
\hline Comorbid condition & Number of patients & Percentage \\
\hline HIV & 36 & 21.33 \\
DM & 64 & 7.33 \\
CKD & 22 & 59.33 \\
Cases without any comorbidity & 178 &
\end{tabular}

LLF TB: lower lung field tuberculosis; HIV: human immunodeficiency virus; DM: diabetes mellitus; CKD: chronic kidney disease. Chi-square goodness of fit. $\chi^{2}=200.8, d f=3, P<0.00001$.

Table 3: Diagnostic role of bronchoscopy-guided techniques as compared to conventional sampling methods in LLF TB $(n=170)$

Conventional diagnostic techniques (sputum smear microscopy and induced sputum microscopy for AFB) $60 \quad 110$

Bronchoscopy-guided sampling (BAL and Gene expert MTB/RIF)

155

15

LLF TB: lower lung field tuberculosis. $\chi^{2}=114.17, d f=1, P<0.00001$.

\begin{tabular}{lll}
\hline Table 4: LLF TB in nonresolving pneumonia in study cases diagnosed by bronchoscopic sampling techniques \\
\hline Nonresolving pneumonia cases suspicion of LLF TB $(\boldsymbol{n}=\mathbf{6 0})$ & Positive yield & Negative yield \\
\hline Bronchoscopy-guided bronchial wash & 18 & 42 \\
Bronchoscopy-guided BAL for Gene Xpert & 58 & 02 \\
\hline
\end{tabular}

LLF TB: lower lung field tuberculosis. $\chi^{2}=57.41, d f=1, P<0.00001$. 


\section{Diagnostic yield of conventional techniques in LLF TB}

In study cases of LLF TB, 57 cases $(20.66 \%)$ were diagnosed by routine sputum microscopic examination for AFB and 80 cases (28\%) were diagnosed by induced sputum microscopic examination for AFB. Our findings are in agreement with previous reports ${ }^{[20,23,24]}$ that bacilli may be difficult to demonstrate on smear or culture and that multiple examinations are often necessary to secure bacteriological proof of TB. Although sputum examination is the simplest way to diagnose LLF TB, isolation of $M$. tuberculosis is difficult on smear or culture examination in no cavitary lesion..$^{\left[{ }^{[]}\right.}$Although sputum microscopy is the most appropriate, low cost, highly specific investigation to diagnose pulmonary TB, in $22-61 \%$ of the cases, sputum smear-negative and culture-positive status is observed. ${ }^{[27]}$ Causes of smear negativity include low bacterial load (less than 10,000 bacilli/ml), poor quality of sputum sample (submitting saliva as sputum), improper preparation and examination of smears, people with late-stage HIV, and immunesuppressed patients and children. ${ }^{[26]}$

Contradictory to our observation, Pandya et al. ${ }^{\left[{ }^{[5]}\right.}$ reported that the incidence of sputum positivity was higher in LLF TB compared to all cases of pulmonary TB. Zuber Ahmed et al. ${ }^{[26]}$ also observed significantly higher sputum positivity $(65.38 \%)$ in LLF TB than in classical upper lung field TB (48.02\%). Higher bacillary load because of pooling of mucous in lower lung field because of less-efficient expectoration may attribute to higher AFB positivity. ${ }^{[25]}$

Induced sputum examination yields diagnosis in $28 \%$ cases (80/300). In the present study, induced sputum smear positivity is lower compared to previous studies by Saglam et al. ${ }^{[28]}$ with $47 \%$ positivity and Hartung et al. ${ }^{[29]}$ study with $42 \%$ positivity. In another large study of 129 subjects by Mc Williams et al., ${ }^{[30]}$ induced sputum AFB sensitivity was found to be very high, $96 \%$.

\section{Diagnostic yield of bronchoscopy guided techniques in LLF TB}

In study cases of LLF TB, 57 cases $(20.66 \%)$ were diagnosed by routine sputum microscopic examination for AFB done on three samples collected on early morning preferably fasting and 80 cases $(28 \%)$ were diagnosed by induced sputum microscopic examination for AFB. In study of 170 LLF TB cases, bronchoscopyguided samples made highly significant improvement $(P<0.00001)$ in diagnostic yield and made diagnosis in $155(91.17 \%)$ cases.

In the present study, bronchoscopy-guided BAL for AFB yields diagnosis in $32.66 \%$ cases (98/300 cases). Similar yields were documented in studies by Pierrae $e t$ al. ${ }^{[31]}$ as $25 \%$, Bernard DA et al. ${ }^{[32]}$ as $41 \%$, and Khalil KF et al. ${ }^{[33]}$ as $39.53 \%$.

In the present study, sensitivity of Gene Xpert MTB/RIF in BAL specimens in sputum smear-negative LLF TB is 91.17\%. Culture of bronchoscopy sample for MTB was not done because of time factor, cumbersome, and cost effectiveness. Similar yields were documented in studies by Pierrae et al. ${ }^{[31]}$ as $80 \%$, Bernard et al. ${ }^{[32]}$ as $92.3 \%$, Khalil et al. ${ }^{[33]}$ as $91.86 \%$, Lee et al. ${ }^{[34]}$ as $81.6 \%$, and Surendra et al. ${ }^{[35]}$ as $90 \%$, and few other studies ${ }^{[36-39]}$ from various authors documented sensitivity between $57 \%$ and $75 \%$.

We offered anti-tuberculosis treatment to all BAL Gene Xpert MTB/RIF negative cases also in "high index of suspicion" scenario, and final treatment outcome as "cure" was considered as confirmatory to document "case of pulmonary TB" not diagnosed by rapid nucleic acid amplification test.

In study of 170 LLF TB cases, head-to-head comparison between conventional diagnostic techniques (sputum microscopy and induced sputum microscopy for AFB) made diagnosis in 60 cases, while bronchoscopy-guided sampling techniques (BAL for $\mathrm{AFB}$ and $\mathrm{BAL}$ for Gene Xpert MTB/RIF) made diagnosis in 155 cases. Bronchoscopy-guided samples are found to be highly significant $(P<0.00001)$ and superior to conventional diagnostic techniques. This is our first study to document comparison of conventional and interventional endoscopyguided newer tests to diagnose LLF TB.

Analyzing post hoc by odds ratio suggests that bronchoscopy-guided sampling techniques (bronchial wash for AFB and BAL for gene Xpert MTB/RIF analysis) is 18.94 times superior to conventional investigation techniques (sputum smear microscopy and induced sputum microscopy for AFB) available to diagnose LLF TB.

\section{LLF TB in non-resolving pneumonia, and role of bronchoscopy in these cases}

In study of 300 LLF TB cases, 60 cases $(20 \%)$ were having NRP pattern. In LLF TB cases with NRP pattern, bronchoscopy-guided bronchial wash microscopy for AFB made diagnosis in 18 cases (42\%), while bronchoscopyguided BAL for Gene Xpert MTB/RIF made diagnosis in 58 cases $(96.66 \%)$. BAL Gene Xpert MT/RIF analysis is highly significant $(P<0.00001)$ and superior to BAL microscopy for AFB. Choudhury et al. ${ }^{[40]}$ observed TB as etiological factor in $16.67 \%$ cases $(10 / 60)$ and all those were diagnosed by bronchoscopy-guided techniques. Silver et al. ${ }^{[41]}$ found tuberculosis in $5.7 \%$ cases from culture of BAL fluid as a cause of NRP. 


\section{Prevalence of comorbidities in LLF TB}

In study cases of LLF TB, co-morbid conditions such as HIV coinfection in 36 cases $(12.00 \%)$, diabetes mellitus in 64 cases $(21.33 \%)$, and CKD in 22 cases $(7.33 \%)$ were observed $(P<0.00001)$. Studies ${ }^{[3,25,42]}$ have shown higher incidence of LLF TB in diabetics.

\section{Other Important observations \\ Gene Xpert in LLF TB}

Analyzing post hoc by odds ratio suggests that analysis using bronchoscopy-guided sample techniques including BAL for Gene Xpert MTB/RIF is 49.71 times superior to bronchial wash and also conventional investigation methods available to diagnose LLF TB in cluster of NRP cases.

\section{Culture methods are not used for the diagnosis of LLF TB}

Rapid culture methods for MTB were not available in our hospital. Conventional or LJ media was available, but because of more cumbersome and time-consuming results of solid media, we avoided to use and enroll in our study. As yield of nucleic acid amplification tests such as gene Xpert MTB/RIF is satisfactory and comparable with culture methods in all sputum-negative cases and excellent in sputum smear-negative bronchial wash specimens, we recommend BAL Gene Xpert as "gold standard" for lower lung field smear-negative TB. These tests are rapid and sensitive even in BAL specimens in LLF TB cases.

\section{Pregnancy and LLF TB}

Pregnancy is an additional risk factor for LLF TB and documented in 9 cases in our study. Although pregnancy increases the risk of acquiring $\mathrm{TB}$ during the course of gestation, LLF TB is common as compared to "classical upper lung field" TB. Although exact prevalence and incidence is not known, few studies ${ }^{[6,15,42]}$ also show increased risk of LLF TB in pregnant females.

\section{Empirical ATT in LLF TB in high index of suspicion scenario}

TB should be considered a diagnostic possibility in patients with "lower lung field pneumonia" positive for constitutional symptoms and negative for Gene Xpert MTB/RIF. The response to the treatment with antituberculosis drugs was excellent with complete resolution of radiological opacities and clinical recovery in our study. All study cases were declared as "cured and treatment completed."

\section{CONCLUSIONS}

LLF TB is usually underdiagnosed because of the diverse clinical and radiological presentation. LLF TB is missed routinely due to less diagnostic yield of conventional diagnostic modalities, and these modalities are used routinely and universally.

Bronchoscopy-guided diagnostic techniques are superior, sensitive, and reliable to confirm LLF TB. "Gene Xpert MTB/RIF in bronchial wash samples" is found to be the best diagnostic modality in evaluating LLF TB and should be used routinely as "current gold standard."

A proportionate number of NRP cases are having LLF TB and a high index of suspicion is a must while evaluating these cases to have successful treatment outcome.

\section{Conflict of Interest}

None declared.

\section{REFERENCES}

1. WHO, Tuberculosis, WHO, 2015. Available from: http://www.who.int/ mediacentre/factsheets/fs104/en/.

2. Greenberg SD, Frager D, Suster B, Walker S, Stavropoulos C, Rothpearl A, et al. Active pulmonary tuberculosis in patients with AIDS: Spectrum of radiographic findings (including a normal appearance). Radiology 1994; 193:115-9.

3. Segarra F, Sherman DS, Rodriguez-Aguem J: Lower lung field tuberculosis. Am Rev Resp Dis 8737-40, 1963

4. Ossen EZ: Tuberculosis of the lower lobe. N Engl J Med 1844; 230: 693-8.

5. Andosca JB, Foley JA: Basal tuberculosis. J Thorac Surg 1943; 12: 259-66.

6. Pannar MS. Lower lung field tuberculosis. Am Rev Resp Dis 1967; 96: 310-3.

7. Rothstein E. Pulmonary tuberculosis involving the lower lobes. Am Rev Tuberc 1949; 59:39-49.

8. Reisner D. Pulmonary tuberculosis of the lower lobe. Arch Int Med 1965; 56: 258-80.

9. Chang SC, Lee P Y, Perng RP: Lower lung field tuberculosis. Chest 1987; 91: 230-2.

10. Treatment of tuberculosis: guidelines-4th ed. World Health Organization 2010.

11. Blakemore R, Story E, Helb D, Kop J, Banada P, Michelle R. Owens MR, et al. Evaluation of the analytical performance of the Xpert MTB/RIF assay. J Cin Microbiol 2010; 48: 2495-501.

12. Kirtland SH, Winterbauer RH. Slowly resolving chronic and recurrent Pneumonia. Clin Chest Med 1991; 12: 303-18.

13. Lehtomaki K. Clinical diagnosis of pneumococcal adenoviral mycoplasmal and mixed pneumonias in young men. Eur Respir J 1988; 1: 324-9.

14. Kidd P. Basic tuberculous phthisis. Lancet 1886; 2: 616.

15. Mathur KC, Tanwar KL, Razdan JN. Lower lung field tuberculosis. Ind J Chest Dis 1974; 16: 31-41.

16. Vishwanathan R. Tuberculosis of the lower lobe. Brit Med J 1936; 2: 1300.

17. Zuber A, Zaheer MS. Lower lung field tuberculosis-a clinical study. J Indian Acad Clin Med 2003; 4: 116-20.

18. Herbert W. Berger, Margarito G. Gramdo; Lower lung field tuberculosis. Chest 1974: 65: 522-6.

19. Parmar MS. Lower lung field tuberculosis. Am Rev Respir Dis 1967; 95:310-2.

20. Hamilton CE, Fredd H; Lower lobe tuberculosis: A review. JAMA 1935; 105: 427-30.

21. Sharma SK, Mohan A. Lower Lung Field Tuberculosis in textbook of Tuberculosis. New Delhi: Jaypee Brothers India 2001; pp. 159-62. 
22. S. Satya Sri. Textbook of pulmonary and extra pulmonary tuberculosis. 3rd ed. New Delhi: Interprint publication 1998; pp. 120.

23. Sokoloff MJ. Lower lobe tuberculosis. Radiology 1940; 34: 589-94.

24. Gordon BL, Cham R, Sokoloff MJ. Basal pulmonary tuberculosis: results of treatment. Am Rev Tuberc 1944; 49: 432-6.

25. Pandya L, Al-Sharif N, Maraey MA. Lower lung field tuberculosis in Saudi Arabia. An Saud Med 1990; 4: 374-7.

26. Ahmad Z, Shoaib Zaheer M. Lower Lung Field Tuberculosis-A Clinical Study. JIACM 2003; 4: 116-20.

27. Toman $\mathrm{K}$. What are the main causes of false-positive and false-negative sputum smears? In: Frieden T, editor. Toman's Tuberculosis Case Detection, Treatment and Monitoring: Questions and Answers; 2nd ed. Geneva: World Health Organization, 2004: pp. 23-27.

28. Saglam L, Akgun M, Aktas E. Usefulness of induced sputum and fibreoptic bronchoscopy specimens in the diagnosis of pulmonary tuberculosis. J Int Med Res 2005; 33: 260-5.

29. Hartung TK, Maulu A, Nash J, Fredlund VG. Suspected pulmonary tuberculosis in rural South Africa - sputum induction as a simple diagnostic tool? S Afr Med J 2002; 92: 455-8.

30. McWilliams T, Wells AU, Harrison AC, Lindstrom S, Cameron RJ, Foskin E. Induced sputum and bronchoscopy in the diagnosis of pulmonary tuberculosis. Thorax 2002; 57: 1010-4.

31. Pierre Le Palud P, Cattoir V, Malbruny B, Magnier R, Campbell K, Oulkhouir $Y$, et al. Retrospective observational study of diagnostic accuracy of the Xpert ${ }^{\oplus} \mathrm{MTB} / \mathrm{RIF}$ assay on fiberoptic bronchoscopy sampling for early diagnosis of smear-negative or sputum-scarce patients with suspected tuberculosis. BMC Pulm Med 2014;14: 137.

32. Barnard DA, Irusen EM, Bruwer JW, Plekker D, Whitelaw AC, Deetlefs JD, et al. Koegelenberg The utility of Xpert MTB/RIF performed on bronchial washings obtained in patients with suspected pulmonary tuberculosis in a high prevalence setting. BMC Pulm Med 2015; 15: 103.

33. Khalil KF, Butt T. Diagnostic yield of bronchoalveolar lavage gene xpert in smear-negative and sputum-scarce pulmonary tuberculosis. J Colle Physicians Surg Pak 2015; 25: 115-8.

34. Lee HY, Seong MW, Park SS, Hwang SS, Lee J, Park YS, et al. Diagnostic accuracy of Xpert ${ }^{\circ}$ MTB/RIF on bronchoscopy specimens in patients with suspected pulmonary tuberculosis. Int J Tuberc Lung Dis 2013;17: 917-21.

35. Sharma SK, Kohli M, Yadav RN, Chaubey J, Bhasin D, Sreenivas V, et al. Evaluating the diagnostic accuracy of xpert $\mathrm{mtb} / \mathrm{rif}$ assay in pulmonary tuberculosis. Available from: http://journals.plos.org/plosone/ article?id=10.1371/journal.pone.0141011. Accessed on September 4, 2017.

36. Norin J. A retrospective evaluation study of diagnostic accuracy of Xpert $^{\oplus}$ MTB/RIF assay, used for detection of Mycobacterium tuberculosis in Greece. Available from: http://www.diva-portal.se/smash/get/ diva2:825390/FULLTEXT01.pdf. Accessed on September 4, 2017.

37. Zeka AN, Tasbakan S, Cavusoglu C. Evaluation of the GeneXpert MTB/ RIF assay for rapid diagnosis of tuberculosis and detection of rifampin resistance in pulmonary and extrapulmonary specimens. J Clin Microbiol 2011; 49: 4138-41.

38. Armand S, Vanhuls P, Delcroix G, Courcol R, Lemaître N. Comparison of the Xpert MTB/RIF test with an IS6110-TaqMan real-time PCR assay for direct detection of Mycobacterium tuberculosis in respiratory and nonrespiratory specimens. J Clin Microbiol. 2011; 49: 1772-6.

39. Zmak L, Jankovic M, Katalinic Jankovic V. Evaluation of Xpert MTB/RIF assay for rapid molecular diagnosis of tuberculosis in a two-year period in Croatia. Available from: http://www. sciencedirect.com/science/article/ pii/S2212553113000538. Accessed on September 4, 2017.

40. Chaudhuri AD, Mukherjee S, Nandi S, Bhuniya S, Tapadar SR, Saha M. A study on non-resolving pneumonia with special reference to role of fiberoptic bronchoscopy. Lung India 2013; 30: 27-32.

41. Feinsilver SH, Fein AM, Niederman MS, Schult DE, Faegenburg DH. Utility of fiberoptic bronchoscopy in non-resolving pneumonia. Chest 1990; 98: 1322-6.

42. Ramachandra, Jithendranath, Agarwal GN, Srivastava VK, Mukerji PK; Lower lung field tuberculosis. Ind J Tub 1982; 29: 158-72.

How to cite this article: Patil S, Narwade S, Mirza M. Bronchial wash Gene Xpert MTB/RIF in lower lung field tuberculosis: Sensitive, superior, and rapid in comparison with conventional diagnostic techniques. J Transl Intern Med 2017; 5: 174-85. 